\title{
Risiken bannen, Chancen wahrnehmen
}

\section{VON JONAS FÜNFGELD, \\ THOMAS SCHWARZ UND \\ BERND SCHWIEN}

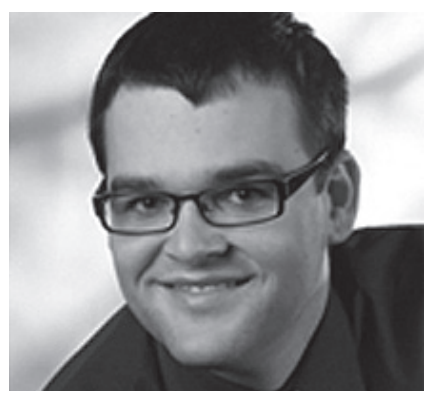

Jonas Fünfgeld studierte an der Fachhochschule Nordhausen mit dem Abschluss Sozialmanager (B.A.).

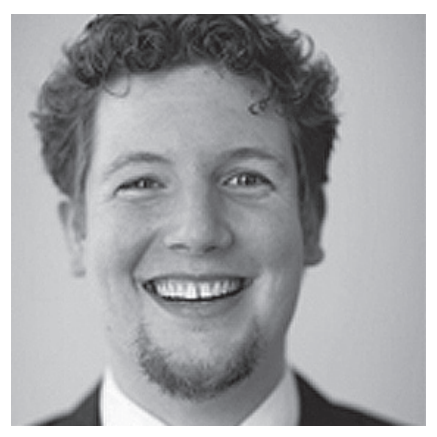

Thomas Schwarz leitet im Diakonischen Werk Schweinfurt e. V. die Abteilung Controlling, Einkauf und IT.

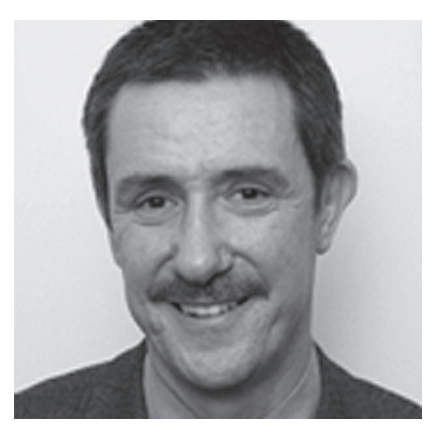

Prof. Dr. Bernd Schwien lehrt an der Fachhochschule Nordhausen Sozialmanagement mit dem Schwerpunkt »Finanzierung sozialwirtschaftlicher Organisationen«.

E-Mail schwien@fh-nordhausen.de

\author{
Eine verantwortungsvolle Unternehmensführung \\ kann durch ein organisationsspezifisches \\ Risikomanagementsystem entscheidend unterstützt \\ werden. Wie ein solches Konzept auch in kurzer Zeit \\ formuliert und einführungsreif erstellt werden kann, \\ zeigt das Beispiel des Diakonischen Werkes Schweinfurt.
}

In einem immer komplexer werdenden ökonomischen, gesellschaftlichen, politischen, technologischen und ökologischen Umfeld steigen die Informationsbedürfnisse der Anspruchsgruppen sozialwirtschaftlicher Organisationen (Stakeholder) nach Transparenz und einbindender Unternehmensführung.

Gerade die sozialwirtschaftlichen Unternehmen müssen sich den steigenden Anforderungen an eine gute und verantwortungsvolle Unternehmensführung, die Instrumente integriert und eine mehrdimensionale, möglichst ganzheitliche Betrachtung als Entscheidungsorientierung ermöglicht, in besonders differenzierter Form stellen.

$\mathrm{Zu}$ diesen Anforderungen gehört auch der richtige Umgang mit Risiken. Die Einführung eines Risikomanagementsystems in sozialwirtschaftlichen Unternehmen stellt daher eine logische Schlussfolgerung dar, diesen gestiegenen Anforderungen gerecht zu werden (vgl. Bachert, 2006, S. 52 ff. sowie Schwien, 2009, S. 83 f.). Wie ein Risikomanagementsystem konzeptioniert werden kann, zeigt das Beispiel des Diakonischen Werkes Schweinfurt e. V.

\section{Rechtlicher Hintergrund}

Wenn es um verantwortungsvolle und nachhaltige Unternehmensführung geht, dann ist damit zumeist auch ein funktionierendes Risikomanagementsystem gemeint. Bereits mit dem 1998 verab- schiedeten Gesetz zur Kontrolle und Transparenz im Unternehmensbereich (KonTraG) wurde der Vorstand von börsennotierten Gesellschaften verpflichtet, ein Überwachungssystem einzurichten, das Gefahren frühzeitig erkennt (vgl. Art. 1 KonTraG).

Mit dem Deutschen Corporate Governance Kodex (DCGK) gibt es seit 2002 ein Regelwerk, das gesetzliche Vorschriften und anerkannte Standards guter und verantwortungsvoller Unternehmensführung enthält. Der Deutsche Corporate Governance Kodex richtet sich in erster Linie an börsennotierte Gesellschaften, eine Beachtung wird aber auch nicht börsennotierten Gesellschaften ausdrücklich empfohlen.

Der Deutsche Corporate Governance Kodex verpflichtet den Vorstand ausdrücklich, für ein angemessenes Risikomanagement und Risikocontrolling im Unternehmen zu sorgen (vgl. Nr. 4.1.4 DCGK). Trotz des Gesetzes zur Kontrol-

"Der Vorstand hat geeignete Maßnahmen zu treffen, insbesondere ein Überwachungssystem einzurichten, damit den Fortbestand der Gesellschaft gefährdende Entwicklungen früh erkannt werden."

§ 91 Abs. 2 des Aktiengesetzes

»Der Vorstand sorgt für ein angemessenes Risikomanagement und Risikocontrolling im Unternehmen."

Punkt 4.1.4 des Deutschen Corporate Governance Kodex 
le und Transparenz im Unternehmensbereich und dem Deutschen Corporate Governance Kodex ergibt sich für eine nicht börsennotierte Gesellschaft, wie das Diakonische Werk Schweinfurt, keine rechtlich bindende Verpflichtung, ein Risikomanagementsystem einzuführen. Dennoch ist es für sozialwirtschaftliche Unternehmen empfehlenswert, Entwicklungen, die sich auf den Unternehmenserfolg auswirken, frühzeitig zu erkennen und im Blick zu behalten. Deshalb hat sich das Diakonische Werk Schweinfurt für die Einführung eines Risikomanagementsystems entschieden (vgl. Diakonischer Corporate Governance Kodex, Stand: Oktober 2005, S. 454; in Bachert, 2006).

\section{Ausgangslage}

Das Diakonische Werk Schweinfurt e. V. ist ein Komplexträger mit mehr als 500 hauptamtlichen Mitarbeiterinnen und Mitarbeitern und einem Jahresumsatz von über 20 Millionen Euro. Über Geschäftsbesorgungsverträge ist das Diakonische Werk Schweinfurt zudem mit anderen diakonischen Werken in der Region verbunden, so dass Einrichtungen der stationären und ambulanten Altenhilfe, der Kinder- und Jugendhilfe, der Behindertenhilfe, ebenso wie zahlreiche Sozialen Dienste und Beratungsangebote zentral vom Diakonischen Werk Schweinfurt betreut werden.

Die Konzipierung eines modularen, also eines aus mehreren Teilelementen bestehenden Risikomanagementsystems, angepasst an die bestehenden Strukturen eines vielseitigen und vielschichtigen Komplexträgers, ist eine dementsprechend anspruchsvolle Aufgabe. Anspruchsvoll war auch der zeitliche Rahmen: Das Risikomanagementsystem sollte innerhalb von drei Monaten entwickelt sein. Das Ziel war es, das Risikomanagementsystem in der Handhabung so einfach wie möglich zu gestalten und den erforderlichen Aufwand möglichst gering zu halten. Eine Mitgestaltung und Entscheidungsorientierung aller Leitungsebenen stand dabei im Vordergrund.

\section{Risikopolitik}

»In der Praxis zeigt sich, dass jede Aktivität einer Non-Profit-Organisation neben den Risiken auch Chancen mit

\section{Wie ein Risikomanagement in der Praxis funktioniert}

\begin{tabular}{|c|c|c|c|}
\hline \multicolumn{4}{|c|}{ Risikofaktoren im Bereich Wirtschaftlichkeit } \\
\hline Risikofaktor & \begin{tabular}{|l|} 
Eintrittswahr- \\
scheinlichkeit \\
Gefahr \\
\end{tabular} & $\begin{array}{l}\text { Ausmaß } \\
\text { Gefahr }\end{array}$ & Beschreibung der Gefahr \\
\hline $\begin{array}{l}\text { Gibt es Risiken im Bereich von } \\
\text { Anschaffungen und } \\
\text { Investitionen? }\end{array}$ & 5 & & \begin{tabular}{|l} 
Kostensteigerung um 85\% im \\
Rahmen einer \\
4
\end{tabular} \\
\hline
\end{tabular}

Der praktische Einsatz des Risikomanagementsystems im Diakonischen Werk Schweinfurt kann an einem fiktiven Fallbeispiel gezeigt werden.

Im Bereich der Wirtschaftlichkeit wird nach Risiken im Zusammenhang mit Anschaffungen und Investitionen gefragt. Die Einrichtungsleitung beurteilt in diesem Fall Eintrittswahrscheinlichkeit und Ausmaß von Chancen und Gefahren, die mit Anschaffungen und Investitionen im Zusammenhang stehen.

Wenn hier nun unplanmäßig höhere Investitionen nötig wären - beispielsweise könnten im Laufe einer Sanierungsmaßnahme verdeckte Baumängel zu einer Kostensteigerung von 85 Prozent führen - dann würde die Einrichtungsleitung diese konkrete Risikolage beschreiben und bewerten. Wenn die Eintrittswahrscheinlichkeit beispielsweise mit fünf Punkten und das Ausmaß (signifikante Schadenshöhe) mit vier von fünf Punkten bewertet werden würde, dann läge der Gefahrenwert mit 20 Punkten (5 Punkte mal 4 Punkte $=20$ Punkte) oberhalb der individuell gewählten Toleranzgrenze von 15 Punkten.

Nun würde die Einrichtungsleitung zur Angabe einer Steuerungsmaßnahme aufgefordert werden, in diesem Fall könnte dies beispielsweise die Prüfung einer Nachfinanzierung durch den Kostenträger oder ein Bankdarlehen sein.

Die Geschäftsbereichsleitung und der Vorstand erfahren über die Risikoübersicht auf Geschäftsbereichsebene den Gefahrenwert und können die vorgeschlagene Steuerungsmaßnahme einsehen. Die konkrete Gefahr von höheren Sanierungskosten, aufgrund von Baumängeln, wäre nun also auf Einrichtungs-, Geschäftsbereichs- und Vorstandsebene bekannt, ebenso wie die entsprechende Steuerungsmaßnahme.

Die Gefahr wird dadurch bewusst wahrgenommen, Steuerungsmaßnahmen und ihre Wirkungen werden permanent beobachtet.

Jonas Fünfgeld, Thomas Schwarz, Bernd Schwien

sich bringt.« (Bachert/Peters/Speckert, 2008, S. 23). Umfasst der Risikobegriff im engeren Sinn lediglich die Gefahr eines Verlustes, so ist unter einem Risiko im weiteren Sinn eine potenzielle Zielabweichung zu verstehen.

Diese Zielabweichung kann positiv oder negativ sein. Demnach sind unter einem Risiko im weiteren Sinn sowohl Gefahren als auch Chancen zu verstehen (vgl. Lück/Unmuth, 2006, S. 16). Da mit dem Risikomanagementsystem des Diakonischen Werkes Schweinfurt sowohl Gefahren als auch Chancen erfasst werden, ist dem Risikomanagementsystem ganz bewusst der erweiterte Risikobegriff zugrunde gelegt worden.

"Nichts geschieht ohne Risiko - aber ohne Risiko geschieht auch nichts." (Keitsch, 2004, S. 1). Dieses Zitat verdeutlicht, dass Risiken zu unternehmerischem Handeln gehören. Unter Risikomanagement ist somit keine reine
Risikovermeidung, sondern vielmehr der bewusste, transparente und verantwortungsvolle Umgang mit Risiken zu verstehen.

Diese Aussage entspricht auch der Risikopolitik des Diakonischen Werkes Schweinfurt. Die formulierten Ziele des Risikomanagements sind:

- Sicherung der Existenz des Diakonischen Werkes Schweinfurt e. V. und der angeschlossenen Träger

- Sicherung des zukünftigen Erfolgs

- Vermeidung bzw. Senkung der durch Gefahren verursachten Kosten und

- Steigerung des Marktwerts des Diakonischen Werkes Schweinfurt

Es wird explizit darauf hingewiesen, dass die vollständige Beseitigung aller Unternehmensgefahren nicht Ziel des Risikomanagements sein kann, da eine derartige Risikopolitik auch die Verfol- 
gung von Chancen vielfach verhindern würde.

\section{Aufbau des Risiko- managementsystems}

Das Risikomanagementsystem des Diakonischen Werkes Schweinfurt ist modular aufgebaut und gliedert sich in die Bereiche Controlling, Frühaufklärungssystem und Internes Überwachungssystem (vgl. Abb. 1). Das Frühaufklärungssystem dient der rechtzeitigen Wahrnehmung von Chancen und Gefahren. Das fundamentale Element des Frühaufklärungssystems stellt hierbei der Risikokatalog dar. Dieser ist unterteilt in drei Bereiche Wirtschaftlichkeit, Leistungserbringung und Umfeld.

Die einzelnen Bereiche sind nochmals in jeweils acht bis zehn Teilbereiche unterteilt, wobei hier auf eine gezielte Nennung von Einzelrisiken verzichtet wird. Vielmehr wird ein bestimmter Teilbereich genannt, in dem Einzelrisiken vorhanden sein können. Eine exemplarische Aufzählung verdeutlicht die möglichen Einzelrisiken des Teilbereichs. Dadurch gelingt es den Risikokatalog relativ kurz

\section{Risikomanagementsystem}

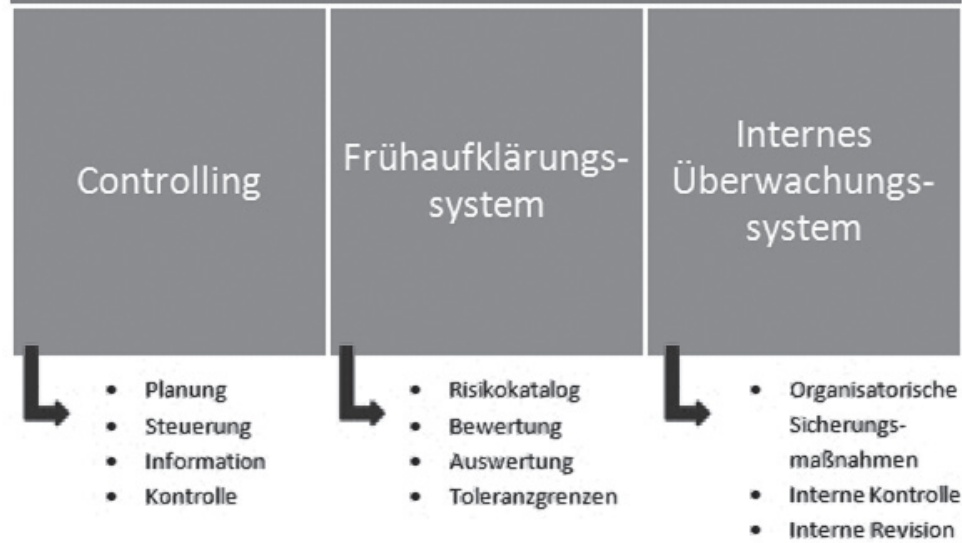

Abb. 1: Das Risikomanagementsystem des Diakonischen Werkes Schweinfurt ist modular aufgebaut.

diesem Fall nach einem Risiko im Bereich der Qualität der Dienstleistung gefragt, wobei die Benotung durch den Medizinischen Dienst der Krankenkassen als eines von mehreren Beispielen für ein potenzielles Einzelrisiko in diesem Teilbereich aufgelistet ist.

\section{"In einem weiten Verständnis bezeichnet ,Risiko eine Planabweichung - meint also sowohl Gefahren wie Chancen"}

und übersichtlich zu halten, ohne Einzelrisiken komplett außen vor zu lassen (vgl. Abb. 2).

Der Risikokatalog gilt für alle Einrichtungen des Diakonischen Werkes Schweinfurt e. V. Eine vollständige Auflistung aller Einzelrisiken wäre nicht nur deutlich aufwendiger, es wäre auch notwendig, für jeden Geschäftsbereich eine eigene Auflistung zu erstellen. Beispielsweise kann die Benotung der Pflegeeinrichtungen durch den Medizinischen Dienst der Krankenkassen im Bereich der Altenhilfe durchaus ein Risiko darstellen, im Bereich der Kinder- und Jugendhilfe würde die Abfrage dieses Einzelrisikos jedoch einen vermeidbaren Zusatzaufwand darstellen, da hier keine Benotung durch den Medizinischen Dienst der Krankenkassen erfolgt. Mit der Abfrage von Teilbereichen wird in
Durch eine Vielzahl von Gesprächen mit Mitarbeitenden aus allen Geschäftsbereichen und Hierarchieebenen wurde sichergestellt, dass der Risikokatalog die reale Risikosituation der verschiedenen Einrichtungen erfasst und abbildet.

\section{Risikokatalog}

\begin{tabular}{|l|l|l|}
\hline Finanzen & Leistungserbringung & Umfeld \\
\hline $\begin{array}{l}\text { Anschaffungen, Investitionen } \\
\text { (z.B. Bäderausstattung, Anbau, } \\
\text { PKW, Büroausstattung) }\end{array}$ & $\begin{array}{l}\text { Personal (2.B. P-Entwicklung, } \\
\text { P-Deckung, P-Qualifikation) }\end{array}$ & Wettbewerbssituation \\
\hline $\begin{array}{l}\text { Auslastung (z.B. Kundenanzahl, } \\
\text { Belegquote, Kundenstruktur) }\end{array}$ & $\begin{array}{l}\text { Unternehmenskultur (Umgang } \\
\text { mit Konflikten, Arbeitsklima, } \\
\text { Fluktuation, Zufriedenheit der } \\
\text { Mitarbeitenden, MAV) }\end{array}$ & $\begin{array}{l}\text { Staatliche Einflüsse (Gesetze, } \\
\text { Richtlinien, Reformen, } \\
\text { Sozialausgaben, } \\
\text { Versorgungsverträge) }\end{array}$ \\
\hline$\vdots$ & $\vdots$ & $\vdots$ \\
\hline
\end{tabular}

Abb. 2: In einem Risikokatalog können beispielhaft mögliche Risiken - und Chancen aufgezählt werden.

\section{Ablauf des Risikomanagements}

Das Risikomanagement besteht aus mehreren, aufeinander aufbauenden Prozessen, die zyklisch durchlaufen werden (vgl. Abb. 3). Die Identifikation der Risiken erfolgt durch den Risikokatalog.

Nach dieser Auflistung werden die Risiken mit einem excelbasierten Fragebogen erfasst und bewertet. Die $\mathrm{Ab}$ frage erfolgt dabei getrennt nach Chancen und Gefahren, wobei jeweils alle Teilbereiche nach potenziellen Risiken abgefragt werden. Jeder Teilbereich wird hinsichtlich der Eintrittswahrscheinlichkeit von Risiken und dem jeweiligen Risikoausmaß anhand einer Skala von eins bis fünf bewertet. Risikoeintrittswahrscheinlichkeit und Risikoausmaß ergeben miteinander multipliziert den Risikowert.

Ein Risiko kann demnach einen Risikowert zwischen 1 und 25 annehmen, wobei ab einem Risikowert von 15 
Punkten die Toleranzgrenze überschritten ist und Handlungsbedarf signalisiert wird. Das bedeutet, beschriebene Maßnahmen aus dem Risikokatalog zur Risikominimierung oder Risikoumgehung werden eingeleitet, ihre Wirkungen werden regelmäßig (z. B. monatlich) berichtet und bewertet.

Die Auswertung des Fragebogens auf Einrichtungsebene erfolgt automatisch, so dass die jeweilige Einrichtungsleitung direkt im Anschluss an das Ausfüllen des Fragebogens eine grafische Darstellung aller Risiken und eine Auflistung der Risiken oberhalb der Toleranzgrenze erhält.

Die einrichtungsübergreifende Auswertung geschieht in zwei Schritten. Zunächst erhält die jeweilige Geschäftsbereichsleitung eine übersichtliche Darstellung über die Risikowerte der einzelnen Einrichtungen des Geschäftsbereichs, bevor diese samt Kommentar der Geschäftsbereichsleitung an den Vorstand weitergeleitet werden.

Risiken, deren Risikowerte die Toleranzgrenze überschreiten, werden mit Steuerungsmaßnahmen versehen. Ziel einer Steuerungsmaßnahme ist die Gefahrenbewältigung beziehungsweise die Chancenergreifung. Gefahren und Chancen, die sich auf den Unternehmenserfolg auswirken würden, sollen dadurch aktiv gesteuert werden. Dies ist zwar nicht immer möglich, manche Gefahren müssen auch einfach akzeptiert werden, andere hingegen können in Eintrittswahrscheinlichkeit oder Ausmaß gesenkt werden.

Bei Chancen hingegen lässt sich eventuell Eintrittswahrscheinlichkeit oder Ausmaß erhöhen. Die Einrichtungsleitung erhält deshalb nach dem Ausfüllen des Fragebogens eine Liste mit Gefahren und Chancen oberhalb der Toleranzgrenze von 15 Punkten mit der Möglichkeit, direkt Steuerungsmaßnahmen anzugeben. Diese Liste wird auch der jeweiligen Geschäftsbereichsleitung zugänglich gemacht, so dass ein einrichtungsübergreifender Vergleich der Steuerungsmaßnahmen möglich ist. Die Verantwortung für den richtigen Umgang mit Risiken verteilt sich auf verschiedene Hierarchieebenen und das Lösungsfindungspotenzial ist nicht auf die oberste Ebene begrenzt.

Durch eine quartalsmäßige Erfassung der Risiken ist eine frühzeitige Reaktion auf Risiken ebenso gesichert, wie

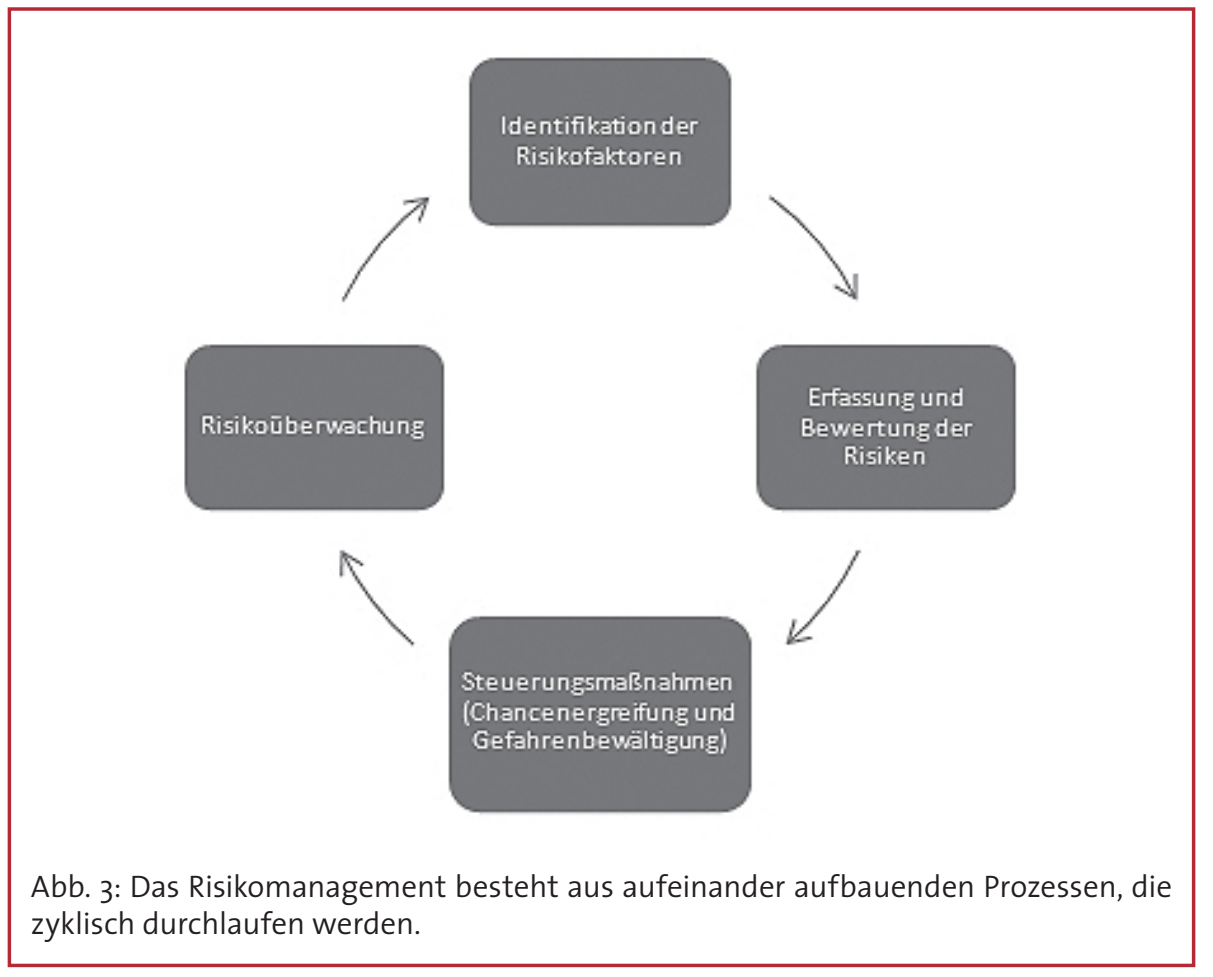

die kontinuierliche Überprüfung des Erfolgs der Steuerungsmaßnahmen. Die Risikoentwicklung lässt sich durch einen Vergleich mit den vorhergehenden Auswertungen darstellen.

\section{Fazit}

Die Notwendigkeit, für ein funktionales Risikomanagementsystem zur Sicherstellung des Fortbestands und zur nachhaltigen Wertschöpfung ist auch in sozialwirtschaftlichen Unternehmen gegeben. Die Konzipierung eines passgenauen Risikomanagementsystems, das die vielfältigen Anforderungen eines Komplexträgers erfüllt, war unter den gegebenen, sehr guten Bedingungen innerhalb von zweieinhalb Monaten

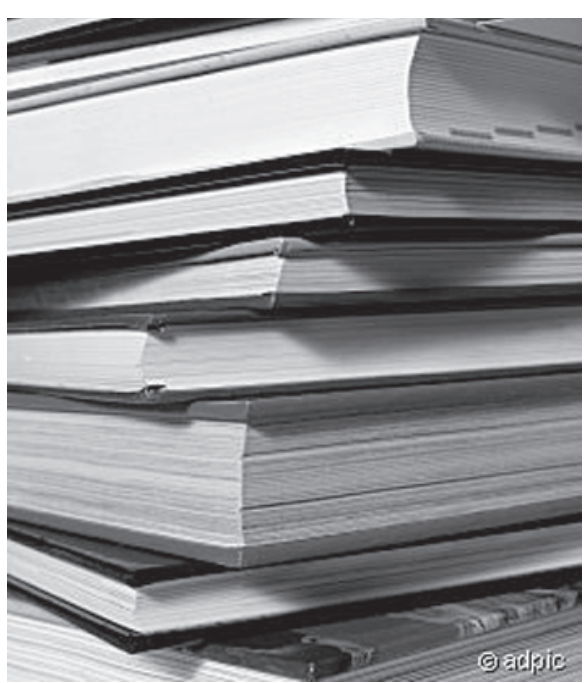

möglich. Insbesondere die Fokussierung auf eine möglichst einfache Handhabung und einen vertretbaren Aufwand haben zu einem schlanken und eleganten Gesamtpaket geführt, das dennoch eine Vielzahl an Möglichkeiten bietet. Durch den modularen Aufbau des Risikomanagementsystems wird eine gute Anpassung an bestehende Strukturen ebenso sichergestellt wie die notwendige Flexibilität.

\section{Literatur}

Bachert, Robert (Hg.), Corporate Governance in Nonprofit-Unternehmen, wrs-Verlag, München 2006.

Schwien, Bernd, Ganzheitliche Unternehmensführung in Nonprofit-Organisationen, Schäffer/ Poeschel, Stuttgart 2009.

KonTraG - Gesetz zur Kontrolle und Transparenz im Unternehmensbereich.

DCGK - Deutscher Corporate Governance Kodex.

Lück, Wolfgang/Unmuth, Anja, Interne Revision (IR) und Risikomanagement, In: Lück, Wolfgang (Hg.), Zentrale Tätigkeitsbereiche der Internen Revision. Aktuelle und zukünftige Schwerpunkte erfolgreicher Revisionsarbeit, Erich Schmidt Verlag GmbH \& Co., Berlin 2006. Keitsch, Detlef, Risikomanagement, 2. Auflage, Schäffer-Poeschel, Stuttgart 2004.

Bachert, Robert/Peters, André/Speckert, Manfred (Hg.), Risikomanagement in sozialen Unternehmen, Nomos, Baden-Baden 2008. 